\title{
Molecular Dynamic Simulation of Thymol Release from PVA-based Antibacterial Film under Different Temperature
}

\author{
Xian-Tao Zhang, Meng-Ya Li, Xiu-Ling Huang \\ School of Mechatronics Engineering and Automation, \\ Shanghai University, \\ Shanghai, China \\ E-mail:xiulh@shu.edu.cn
}

\author{
Xiu-ling Huang \\ Jiangsu Key Laboratory of Advanced Food \\ Manufacturing Equipment \& Technolog , \\ Jiangnan University, \\ Jiangsu, China
}

\begin{abstract}
The release precess and mechanism of thymol from PVA/thymol system is studied based on molecular dynamic simulation under different temperature conditions. PVA/ thymol model of polymer was studied by Molecular dynamics simulation and PVA as the research matrix, then add some necessary antibacterial (thymol). The release process of thymol in polyvinyl alcohol polymer materials was researched under the change of different temperature conditions from three micro-characteristics angles: the mean square displacement, diffusion coefficient and free volume. Results: The free volume fraction of thymol molecules in PVA films will increase with the increase of temperature rising, diffusion coefficient of thymol molecules in PVA films also increases with the increase of temperature rising of polymer film. It also provides a good method for the diffusion about small molecules in polyvinyl alcohol. Conclusion: temperature has an important effect on the release of antimicrobial agents in packaging materials.
\end{abstract}

Keywords-molecular mechanism; temperature

\section{INTRODUCTION}

At present, China's fruit and vegetable antibacterial packaging is mainly facing two problems: one is the loss of two fruits and vegetables; the other is food packaging safety problem is emerging in an endless stream that has caused great harm to the quality of the food safety and consumer health.

Because existing antibacterial fresh-keeping technology mainly rely on passive sterilization antibacterial agent concentration, with the rapid consumption of effective antimicrobial properties, decomposition, antibacterial effect is transient. So a lot of preservatives were directly added to the food in order to achieve good antimicrobial antiseptic effect. High concentrations of antimicrobial agent could not only make bacteria tolerance and also increase the migration of harmful substances into the food, causing some food safety problems [1-4].

Inside the packaging, slow release antibacterial packaging mainly through the slow release of antimicrobials, maintain long-term effective minimum antibacterial concentration, thus attain the goal of antimicrobial antiseptic. A large number of studies have shown that the new antibacterial packaging can not only ensure food safety, but also effectively extend the shelf life of food packing those incomparable advantages than traditional packaging which is a focus of food packaging technology [5-8].

With the rapid development of computer technology, system in the field of molecular dynamics simulation is becoming more widely [9]. Especially in recent years, potential applications of molecular dynamics studying of the migration in the prediction of food packaging safety issues caused more attention from many scholars at home and abroad. Domestic's molecular dynamics study the diffusion process mainly focused on qualitative analysis some factors affecting polymer migration and lack of quantitative analysis the migration dynamic process and mechanism of migration. However, foreign scholars focus on traditionally packing and small molecular compound in the transition and simulation of plastic packaging materials, studying the process of the release of the new type of antimicrobial agent in antimicrobial packaging [10-13]. Polyvinyl alcohol as the research base in the paper by adding thymol, then construct PVA/thymol polymer model. Slow-releasing technology combined with molecular dynamics simulation, from microscopic Angle, and aimed at using the method of molecular dynamics simulation to study the new antimicrobial packaging materials about PVA/thymol under the condition of different temperatures based on release process of antibacterial agent [14-15], then revealed the microscopic mechanism of release to guide the actual production of the new antibacterial slow-release packaging materials, and improve food safety and food quality for important guiding significance.

\section{MD SIMULATION CALCULATION}

\section{A. Modeling and Optimization}

Thymol molecules and 100 repeat unit with end to end in Figure 1 to form a PVA polymer chains was preliminary built, then the energy minimization method is used for structure optimization, the most stable structure can be quickly found, the superposition of the energy in the process of modeling was easily eliminated.

Three-dimensional periodic structure of the Amorphous PVA cellular automata model was built by using the amorphous cell tools, eight thymol molecules were randomly placed in PVA cell to Smart Minimization preliminary optimization of periodic structures. Then 200 ps NVT and NPT simulation and annealing heat treatment was used to obtain equilibrium geometry conformation. 


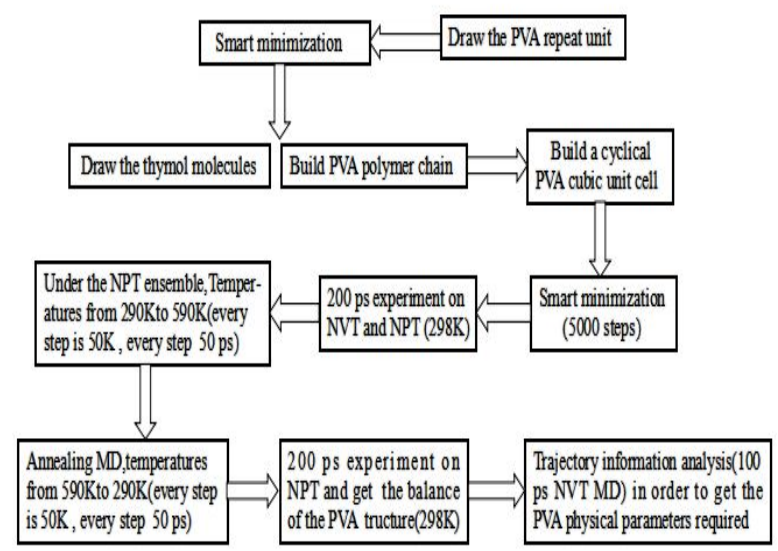

Figure 1. Construction process of PVA/thymol system model.

\section{B. Parameter Settings}

The temperature and pressure control law using the Nose and Berendsen control method in the whole simulation process. Ewald method is used to deal with the key when calculating coulomb interaction energy. Vander Waals force was used by atomic simulation, truncation distance is $9.5 \AA$, spline width is $1.0 \AA$, buffer width is $0.5 \AA$, simulation time step is $1 \mathrm{fs}$, and sampling frequency is 1000 steps in simulation process.

\section{THE RESULTS AND DISCUSSION}

\section{A. Mean Square Displacement}

Use for cite analysis module "Mean Square below" option to obtain optimal equilibrium periodicity of PVA model that temperature respectively are $277 \mathrm{~K}, 298 \mathrm{~K}, 313 \mathrm{~K}$ based on 1000 ps NVT and MD simulation of trajectory curve were analyzed, then obtained all azimuth shift curve under different temperature conditions. Using the Origin of all azimuth shift curve linear fitting by using the linear slope, the slope of the straight line size reflects the activity of the polymer chain.

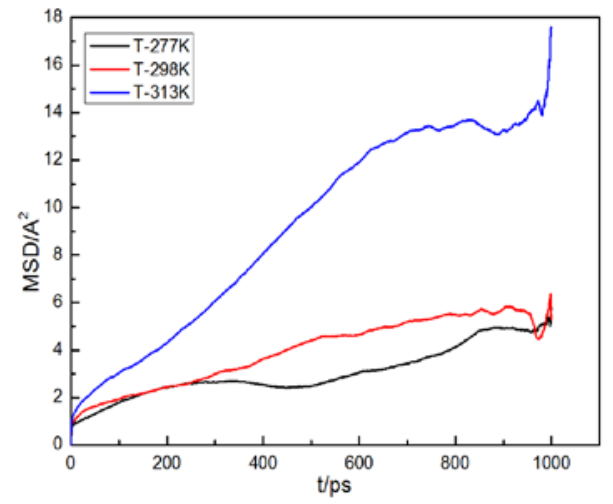

Figure 2. MSD of PVA polymer system under different temperature conditions.

Polymer chain motility of PVA is commonly used according to the MSD curve, azimuth shift curve is more impressive, the motility of molecular chain is stronger.
Azimuth shift curve of PVA polymer system under different temperature conditions in Figure 2.The MSD curve shows that thymol molecule in the PVA polymer system all azimuth shifts will be gradually increased with the rising of temp-erature, obviously the changes of temperature's influence on the diffusion coefficient is bigger. Because temperature will lead to increasing chemical molecular thermal motion, vibration frequency of the cavity will gradually increase, at the same time, polymer chain segment motion of PVA is increasing, inter-atomic forces between chemicals and poly-mer chains is also decreasing, flexible of molecular chain is enhanced, thus more thymol molecule in PVA is released.

\section{B. Free Volume}

Chemicals in the polymer matrix spread is affected by size, shape, number of chemicals, free volume of polymer matrix form and quantity restrictions. Thymol molecules as a probe molecule, Connolly surface method is used to calculate different temperature under the condition of free volume fraction of PVA system. Temperatures respectively are $277 \mathrm{~K}, 298 \mathrm{~K}$ and $313 \mathrm{~K}$. Corresponding free volume distribution as shown in Figure 3, the free volume fraction are shown in tableI.
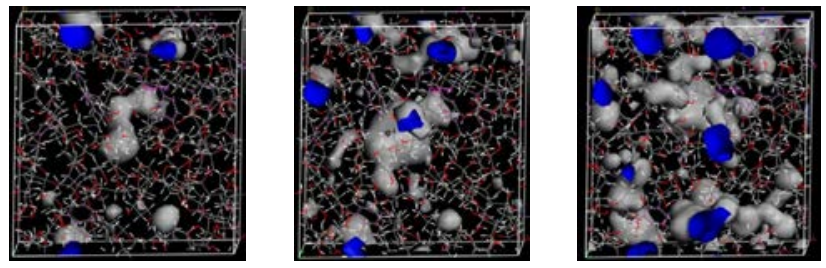

Figure 3. Free volume distribution of thymol in PVA under different temperature conditions.

TABLE I. TABLE FREE VOLUME FRACTION OF THYMOL MOLECULES IN PVA UNDER DIFFERENT TEMPERATURE CONDITIONS

\begin{tabular}{|c|c|c|c|}
\hline \multirow{2}{*}{$\begin{array}{c}\text { Model } \\
\text { name }\end{array}$} & $\mathbf{T}-277 \boldsymbol{K}$ & $\boldsymbol{T}$-298K & $\boldsymbol{T}-313 \boldsymbol{K}$ \\
\cline { 2 - 4 } & $277 \mathrm{~K}$ & $298 \mathrm{~K}$ & $313 \mathrm{~K}$ \\
\hline $\begin{array}{c}\text { Temperat } \\
\text { ure }\end{array}$ & $2.73 \%$ & $2.96 \%$ & $4.20 \%$ \\
\hline $\begin{array}{c}\text { Free } \\
\text { volume } \\
\text { fraction }\end{array}$ & & & \\
\hline
\end{tabular}

Thymol molecule in the PVA system increases as the temperature rises with the free volume fraction in table 1. When the temperature is $313 \mathrm{~K}$, PVA system can be accessed the most free volume hole number, hole size is the largest and the best continuity. The reason is that temperature is rising making PVA chain motility increase, significantly making chemicals and polymer chains interatomic forces decrease, flexible of molecular chain enhance, connecting hole free volume change of short channel frequency, conducive to the free volume accumulation and distribution form.

\section{Diffusion Coefficient}

Relative temperature are respectively $277 \mathrm{~K}, 298 \mathrm{~K}$ and $313 \mathrm{~K}$ in Figure 4, according to the Einsten, diffusion 
coefficient can be obtained by the MSD curve in time. In order to reduce calculation error, general equilibrium are azimuth shift curve as diffusion coefficient calculation basis, MSD and time have a good linear relationship at this time.

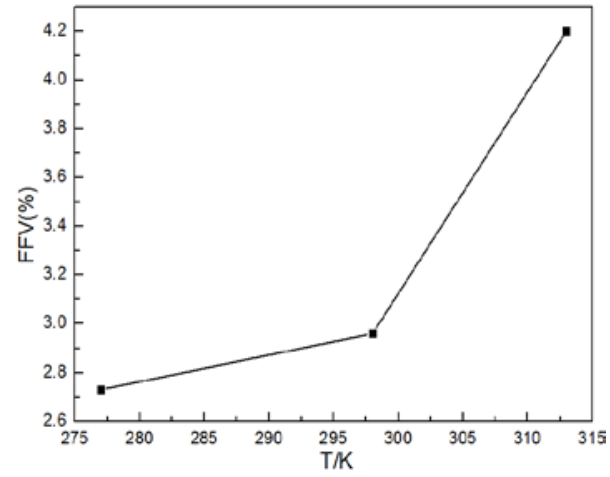

Figure 4. Free volume distribution of thymol in PVA under different temperature conditions.

For the temperature of $277 \mathrm{~K}, 298 \mathrm{~K}$ and $313 \mathrm{~K}$ respectively under thymol molecular diffusion coefficient $\mathrm{D}$ in PVA $\left(10^{-8} \mathrm{~m}^{2} \mathrm{~s}^{-1}\right)$ in table $\Pi$, analysis thymol molecular diffusion coefficient in PVA under different temperature, the conclusion is as follows: 1)Temperature range is $277 \mathrm{~K}$ to $313 K$ (normal atmospheric pressure), thymol molecule in the PVA system is positive correlation. When the conditions are low temperature, the diffusion coefficient curve keep relatively slow growth until reaching at room temperature, diffusion coefficient of growth will increase rapidly. The reason is main due to temperature increase, chemical molecular thermal motion is more stronger, motility and flexible of molecular chain in PVA is enhanced, thus to thymol release in PVA membrane. 2) Normal atmospheric pressure, temperature range are $277 \mathrm{~K}$ to $313 \mathrm{~K}$, thymol molecular diffusion coefficient and the free volume fraction in the PVA system change rule has good consistency.

TABLE II.TABLE DIFFUSION COEFFICIENT OF PVA MODEL UNDER DIFFERENT TEMPERATURE CONDITIONS

\begin{tabular}{|c|c|c|c|}
\hline \multirow{2}{*}{$\begin{array}{c}\text { Model of the } \\
\text { temperature }\end{array}$} & \multicolumn{3}{|c|}{ Model of the temperature } \\
\cline { 2 - 4 } & $\mathbf{2 7 7 K}$ & $\mathbf{2 9 8 K}$ & $\mathbf{3 1 3 K}$ \\
\hline $\begin{array}{c}\text { Rate of } \\
\text { change of } \\
\text { MSD }\end{array}$ & 0.00355 & 0.00469 & 0.01419 \\
\hline $\begin{array}{c}\text { Diffusion } \\
\text { coefficient(D) }\end{array}$ & 0.00059 & 0.00078 & 0.00237 \\
\hline
\end{tabular}

\section{Release Mechanism}

Use Animation Option tools to analyze track file to output the final structure and obtain thymol in PVA system release molecules under different temperatures condition. Figure 5, 6 and 7 are respectively set different temperature conditions: $277 \mathrm{~K}, 298 \mathrm{~K}$ and $313 \mathrm{~K}$ under different simulation time. PVA amorphous thymol molecules in the cell and the model structure of polymer release diffusion motion diagram. The release of thymol molecular trajectory can be obtained: within a longer simulation time, PVA matrix is not rapid diffusion thymol molecular diffusion in PVA, but slow creep [16].

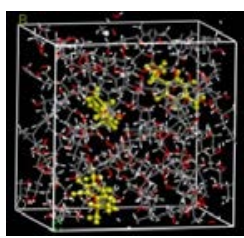

100PS

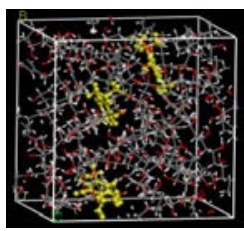

500PS

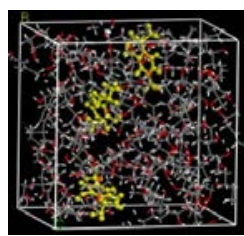

200PS

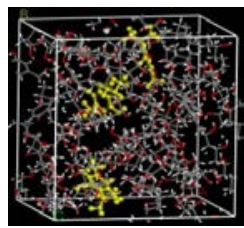

700PS

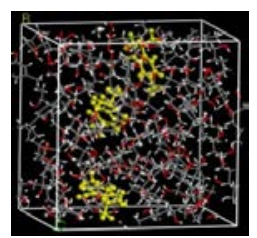

300PS

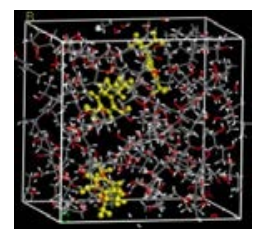

900PS
Figure 5. The motion of thymol molecules in PVA under different simulation time based on 277K.

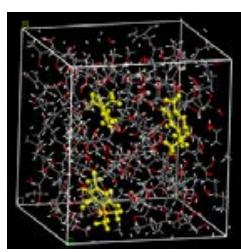

100PS

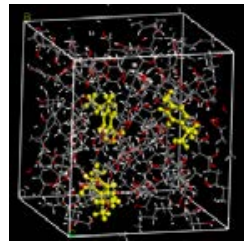

500PS

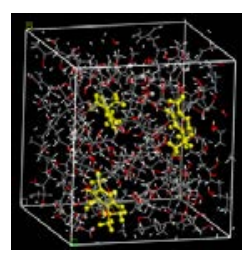

200PS

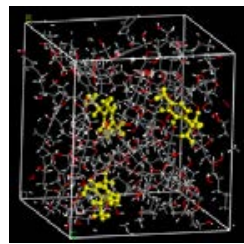

700PS

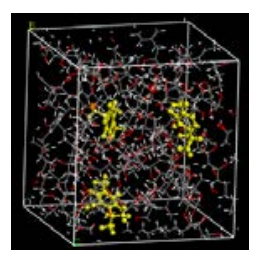

300PS

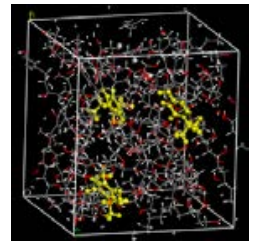

900PS
Figure 6. The motion of thymol molecules in PVA under different simulation time based on $298 \mathrm{~K}$.

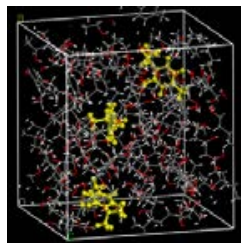

100PS

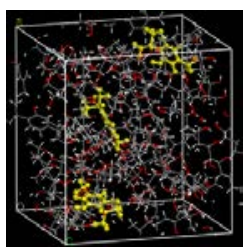

500PS

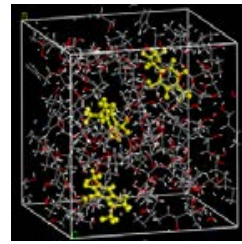

200PS

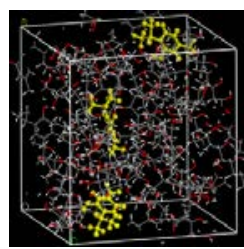

700PS

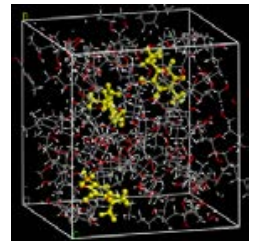

300PS

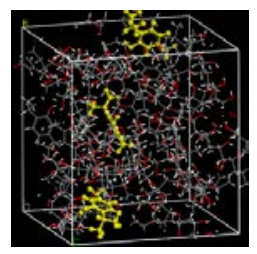

900PS
Figure 7. The motion of thymol molecules in PVA under different simulation time based on $313 \mathrm{~K}$.

\section{CONCLUSION}

The paper mainly studies the simulation of thymol release from PVA under different temperature conditions 
based on compass force field thymol molecule in the PVA/thymol system dynamic release's process, respectively from the free volume fraction, motility of macromolecule chain, four aspects are position shift and diffusion coefficient qualitatively quantitatively analyses the temperature conditions on the antibacterial thymol release process. The release of thymol molecular mechanism from the new type of antibacterial packaging materials in antibacterial agent was revealed based on the microscopic view. The following conclusions: Thymol molecular diffusion coefficient in the PVA polymer system and motility of polymer chain and free volume fraction characteristic has a good consistency. With the increase of temperature, free volume fraction of thymol molecular in the PVA will increase, the diffusion coefficient of thymol will gradually increase, thermal motion of molecules in PVA system gradually strengthened. Chemicals and polymer chains inter-atomic forces, flexible enhancement of molecular chain, which is advantageous to the thymol release in PVA. By observing the thymol molecule in the PVA system and release the moving track, we can find thymol molecules in PVA system for slow creep instead of jumping movement.

\section{ACKNOWLEDGEMENT}

The work is supported by the Opening Fund of Jiangsu Key Laboratory of Advanced Food Manufacturing Equipment \& Technology at Jiangnan University (FM201508).

\section{REFERENCES}

[1] Zhou N, Long J.H, Shen P.Q. Research Status of Preservation Technology of Leafy Vegetables at Home and Abroad [J]. Journal of Anhui Agricultural Sciences, 2013, 41(15): 6897-6899.

[2] Liu Y, Yang X. J. Analysis and Research the Present Situation and Development Trend for fresh-keeping packaging technology [J]. China Packaging, 2001(6): 74-75.
[3] Hotchkiss J H. Food-packaging interactions influencing quality and safety [J]. Food Additives \& Contaminants, 1997, 14(6-7): 601-607.

[4] L Vermeiren, F Devlieghere, M van Beest, N de Kruijf, J Debevere. Developments in the active packaging of foods. Trends in Food Science \& Technology, 1999, 10: 77-86.

[5] LI Q, ZHANG J. Study on food package paper with antibacterial properties [J]. Science and Technology of Food Industry, 2008, 8: 228-230.

[6] Dai H.M, Dai P.H. The cause of food packaging safety hazards analysis and control countermeasures [J]. China Packaging, 2011, (4): 9-13.

[7] M Mastromatteo, M Mastromatteo, A Conte, M A D Nobile. Advances in controlled release devices for food packaging applications. Trends in Food Science \& Technology, 2010, 21: 591598.

[8] P Appending, J H Hotchkiss. Review of antimicrobial food packaging [J]. Innovative Food Science \& Emerging Technologies, 2002, 3: 113-126.

[9] Yang X.Z, Molecular simulation and polymer materials [M]. Beijing: Science Press, 2002.

[10] Hofman D, Fritz L, Ulbrieh J. Molecular simulation of small molecule diffusion and solution in dens amorphous polysiloxanes and polyimides [J]. Comput Theor Polym Sci, 2000, 10: 419-436.

[11] M. Farkhondeh, E. Hossein, M. Jalil. Molecular Dynamics Simulation of Diffusion and Permeation of Gasses in Polystyrene [J]. Polymer, 2010, 51(1): 300-307.

[12] Desai T, Keblinski P, Kumar S K, Molecular dynamics simulation of polymer transport in nanocomposites, J.Chem.Phys., 2005, 122: 1-8.

[13] Wang P.L. Studies on diffusion coefficients of migrants in plastic packaging by molecular dynamics [D]. Jinan University, 2010.

[14] Shi C.P.Research on PVA Slow-release/Controlled-release Packaging Film [D].Hunan University of Technology, 2012.

[15] M Mastromatteo, G Barbuzzi, A Conte, M A D Nobile. Controlled release of thymol from zein based film. Innovative [J]. Food Science and Emerging Technologies, 2009, 10: 222-227.

[16] Xu J, Zhou F, Ji B P, et al. The antibacterial mechanism of carvacrol and thymol against Escherichia coli [J].Letters in applied microbiology, 2008, 47(3): 174-179. 